\title{
Câncer como injustiça social: pesquisa argentina sobre acesso à saúde, diagnóstico, assistência e acompanhamento
}

\author{
Cancer as a social injustice: Argentine research on access to \\ health, diagnosis, assistance, and monitoring
}

Cáncer como injusticia social: investigación argentina sobre acceso

a la salud, diagnóstico, asistencia y seguimiento

IN SITU: EL CÁNCER COMO INJUSTICIA SOCIAL. Luxardo N, Sassetti F, editores. Buenos Aires: Biblos; 2021. 983 p. ISBN: 978-987-691-937.

doi: 10.1590/0102-311X00222721

Em 2021, uma obra de fôlego é lançada na Argentina, intitulada In Situ: El Cáncer como Injusticia Social, com coordenação de Natalia Luxardo \& Fernando Sassetti. Com base na investigação Desafios y Alcances para el Control del Cáncer con Equidade en el Primer Nivel de Atención, os dois pesquisadores do Conselho Nacional de Investigações Científicas e Técnicas da Argentina (CONICET), órgão análogo ao nosso Conselho Nacional de Desenvolvimento Científico e Tecnológico $(\mathrm{CNPq})$, reuniram uma grande equipe, com especialização nas diferentes possibilidades de leitura e entendimento em torno do acesso ao diagnóstico, tratamento e prevenção do câncer - doença conhecida desde a antiguidade. Realizada entre 2016 e 2020, a pesquisa contou com a participação de investigadores da Faculdade de Ciências Sociais da Universidade de Buenos Aires, da Faculdade de Engenharia da Universidade Nacional de Entre Ríos e do Instituto Nacional do Câncer da Argentina.

Para entendimento das perspectivas da obra, faz-se necessário mencionar a formação dos coordenadores da investigação: Natalia Luxardo é graduada em Serviço Social (Universidade de Buenos Aires), mestre em Ciências Sociais com menção em Saúde (Faculdade Latino-Americana de Ciências Sociais), doutora em Ciências Sociais (Universidade de Buenos Aires), doutora em Cultura \& Sociedade (Universidade Nacional de Arte) e pesquisadora do CONICET. Fernando Sassetti é formado em Bioengenharia (Universidade Nacional de Entre Ríos), mestre em Gestão de Sistemas e Serviços da Saúde (Universidade Nacional de Rosario), com doutorado em Engenharia (Universidade Nacional do Litoral). Os diferentes campos de conhecimento da formação dos coordenadores do estudo constituem indicativo da riqueza da abordagem da obra.

A partir de dados de pesquisas prévias e de estudos epidemiológicos que apontaram as diferentes taxas de morbimortalidade do câncer nas distintas regiões da Argentina, a investigação visou responder à seguinte indagação: "qual o papel do nível primário de atenção à saúde e de outros determinantes sociais da saúde nas primeiras fases de controle do câncer em comunidades estruturalmente vulneráveis?”. Diante dessa questão, foram estabelecidos os seguintes objetivos: (1) identificar os desafios e a abrangência do nível primário de atenção à saúde, no que tange ao controle do câncer; (2) analisar como atuam os determinantes das desigualdades sociais associadas à saúde, ao nível local; e (3) descrever a variabilidade do arco de determinantes intermediários da saúde no nível primário de atenção. Como consequência dos dados resultantes das três metas, o estudo apresenta proposições para redução das iniquidades no controle do câncer. 
A investigação se baseou em diferentes referenciais teóricos e metodologias, com base no pressuposto da complexidade das problemáticas vinculadas às iniquidades na saúde e injustiça social. Assim, a perspectiva transdisciplinar foi fundamental para o desenvolvimento da pesquisa, que contou com quatro eixos teóricos. $\mathrm{O}$ primeiro é o campo de estudos conhecido nos Estados Unidos como Antropologia médica interpretativa-crítica, além de contar com outras abordagens sobre o tema, oriundo das Ciências Sociais. A segunda grande área é denominada Saúde Coletiva ou Medicina Social latino-americana; a terceira, Epidemiologia Social de origem anglo-saxã, baseada nos determinantes sociais da saúde e na Ecossociologia. Por fim, o quarto eixo é denominado Epistemologias Colaborativas. A apreensão dos dados ocorreu de diversas formas, a saber: etnografia institucional em locais de atenção primária e documental, em associação com registros audiovisuais e com registros específicos de cada área de atuação profissional, como da geografia, epidemiologia, sociologia, entre outros.

O livro está dividido em 11 seções, além de contar com prefácio do epidemiologista equatoriano Jaime Breilh; prólogo do epistemólogo argentino Claudio Martyniuk; e introdução e conclusões dos organizadores da obra. Com estrutura clara e bem organizada, o panorama do câncer e das desigualdades sociais em saúde é apresentado ao leitor paulatinamente, com um volume significativo de dados históricos, com atualização e contextualização.

Na primeira parte, são apresentados dados sobre as desigualdades e iniquidades da saúde pública na Argentina, com foco sobre o câncer. A segunda parte é dedicada à definição das estratégias para o desenvolvimento do estudo e, na terceira parte, são descritos os modos de vida e de reprodução social nas periferias urbanas. A quarta parte conta com uma apresentação acerca das vulnerabilidades e fortalezas dos territórios investigados, enquanto a quinta seção é dedicada aos processos de deterioração e de proteção de comunidades periurbanas e rurais. A sexta parte contempla os desafios presentes nos estudos em torno do câncer e seus nexos com as questões concernentes ao meio ambiente, como o uso de agrotóxicos. Nos tópicos seguintes são aborda- dos: o sistema de saúde argentino, os sistemas de informação em saúde, a epidemiologia do câncer, as falhas na triagem de casos e, por fim, na décima primeira seção, o uso de recursos artísticos para mobilização de comunidades.

Não há dúvidas acerca da abrangência e profundidade da investigação, com origem em estudo desenvolvido anteriormente por Luxardo. Apresentada em 2013 nas Jornadas Nacionais de Debate Interdisciplinar em Saúde e População no Instituto Gino Germani, a pesquisa foi publicada no livro Cáncer y Sociedad. Múltiples Miradas, Enfoques, Recortes 1.

A investigação na qual se baseou o livro aqui resenhado foi proposta por Luxardo ao Instituto Nacional do Câncer e às instituições parceiras em 2018. O trabalho colaborativo empreendido por todos os participantes da investigação - assistentes sociais, profissionais de saúde, cientistas sociais e artistas - permitiu explicitar como as condições de vida e a estrutura social determinam os processos de saúde/doença em distintos grupos e populações. Para além desses dados, a investigação apontou as estratégias possíveis de enfrentamento e os entraves à prevenção e acesso ao atendimento em saúde.

A obra demonstra, com fartura de dados e em diálogo com referências bibliográficas atuais, os padrões de incidência e de morbimortalidade das doenças entre os distintos grupos populacionais, bem como a centralidade de diferenças por sexo biológico, idade, características genéticas, além da relevância de aspectos sociais e econômicos. Nesse sentido, as taxas de morbimortalidade aumentam em relação direta com a posição social. Assim, as regiões com os melhores indicadores em termos de educação e cobertura formal de assistência em saúde alcançaram reduções na mortalidade por câncer. Portanto, a análise por regiões confirma a inequidade existente no país.

Como não é possível abordar com detalhes as discussões desenvolvidas na obra, nessa resenha apresento o estudo sobre a triagem de mulheres, no que tange ao câncer de colo de útero, no capítulo 'Porque No Quise': Descifrando qué Hay Detrás de los Rechazos o Descontinuidades en los Tamizajes de Cuello de Útero, na décima parte do livro. De acordo com o estudo, as experiências das mulheres investigadas são: (1) as infecções são resolvidas sem atenção médica; (2) conhecimentos das 
formas de intervenção do sistema de saúde; e (3) histórias de outras mulheres possibilitam decisões sobre o que fazer. As resistências à permanência e adesão ao atendimento ambulatorial são várias, desde rechaço às interpelações morais da parte de equipes de saúde sobre o número de filhos, de parceiros sexuais, a idade de início da vida sexual, até a baixa qualidade do atendimento, a burocracia e a falta de vínculo com um profissional de saúde, pela alta rotatividade na unidade - sobretudo ao se tratar das clínicas de certas regiões mais distantes de grandes centros urbanos.

Nas conclusões, os autores afirmam que a construção de sociedades justas, no que concerne à assistência em saúde, depende da busca por entendimento e transformação da tríplice desigualdade, nos termos dos autores: de classe social, etnia e gênero. Sem dúvida, trata-se da possibilidade de implementação, na prática, dos Direitos Humanos. De fato, o controle do câncer se instalou nas agendas globais de atenção à saúde. No entanto, não se trata de buscar tal controle a partir dessa enfermidade. Antes, é preciso garantir amplo acesso ao atendimento em saúde, não somente com foco sobre determinada doença. Por fim, os autores apontam os desafios da construção de justiça e qualidade de assistência em países com desigualdades e iniquidades em saúde, como todos os da América Latina. Sem dúvida, é uma obra de interesse para pesquisadores, gestores e profissionais da área da saúde - sejam aqueles interessados no câncer ou os dedicados a outras enfermidades, uma vez que a investigação empreendida é modelo de investigação a partir de múltiplas perspectivas e abordagens, de transdisciplinaridade e interdisciplinaridade.

\section{Rachel Aisengart}

1 Instituto de Estudos em Saúde Coletiva, Universidade Federal do Rio de Janeiro, Rio de Janeiro, Brasil.

raisengartm@terra.com.br

\section{Informação adicional}

ORCID: Rachel Aisengart (0000-0002-8987-6791).

1. Luxardo N. Cáncer y sociedad. Múltiples miradas, enfoques, recortes. Buenos Aires: Biblos; 2015. 